\title{
Application of Artificial Neural Networking for Determining the Plane of Vibration in Rotating System
}

\author{
Arka Sen ${ }^{1 *}$, Manik Chandra Majumder ${ }^{2}$, Sumit Mukhopadhyay ${ }^{2}$, \\ Robin Kumar Biswas ${ }^{3}$, Subhadeep Roy ${ }^{4}$ \\ ${ }^{1 * 2}{ }^{2}$ Department of Mechanical Engineering, National Institute of Technology Durgapur, West Bengal, India. \\ ${ }^{3}$ Condition Monitoring and Structural Analysis Department, Central Mechanical Engineering Research \\ Institute, Durgapur, India. \\ ${ }^{4}$ Computer Science and Engineering Department, Jalpaiguri Government Engineering College, West Bengal, \\ India.
}

\begin{abstract}
In this paper a new approach for Artificial Neural Networking using Feed Forward Back Propagation Method and Levenberg-Marquardt backpropagation training function has been developed using Java Programming, where by directly feeding the RMS and Phase values of vibration, the unbalance plane can be detected with minimum error. In a Machine Fault Simulator RMS value and phase values of vibrations are collected from the four accelerometers placed in $X$ and $Y$ direction of Left and Right Bearings.Further these data are fed into the neural network for training purpose. In the testing phase of the neural network, the plane of vibration has been determined using different training algorithms available in MATLAB. Their prediction values have been compared with the actual value, errors for different training algorithms are calculated and a conclusion has been drawn for the best training function available for this current research work.
\end{abstract}

Keywords: Condition monitoring, Rotating Equipment, Vibration Analysis, Artificial Neural Networking, MATLAB

\section{Introduction}

In the past few years, the machine condition monitoring has gained much importance in the field of engineering and maintenance. Rotating machines which vibrates can cause catastrophic failures if not attended or maintained properly. Various techniques are available in condition monitoring namely: Vibration Analysis, Oil Debris Analysis, Ferrography, Temperature analysis. Among all these techniques, vibration analysis have gained much importance in the field of condition monitoring because of its accuracy in detecting faults its ability for proper diagnosis of the faults. Vibration-based condition monitoring (VCM) requires vibration measurement on each bearing pedestal using a number of vibration transducers and then signals processing for all the measured vibration data to identify fault(s). Artificial Neural networking technique has gained a lot of importance in the field of predictive maintenance. In the current research work, focus has been given on the establishment of procedure for unbalance plane detection based on the minimum error prediction for different training algorithms.

\section{Literature Review}

Yazhao Qiu (1) presented a fuzzy approach for the analysis of unbalanced nonlinear rotor systems involving uncertain parameters. Jiangping Wang (2) investigated the use of basic fuzzy logic principle as a fault diagnostic technique for five-plunger pump. Fuzzy logic was used to classify frequency spectra according to the likely fault condition which they represent. Javier Sanz (3) in his paper used a combination of the capability of wavelet transform (WT) to treat transient signals with the ability of auto-associative neural networks to extract features of data sets in an unsupervised mode.Yaguo Lei (4) performed fault diagnosis of rotating machinery using statistical analysis, empirical mode decomposition (EMD), adaptive neurofuzzy inference system (ANFIS) and genetic algorithms (GAs). Yaguo Lei (5) used statistical analysis method combined with adaptive neurofuzzy inference system (ANFIS) for fault diagnosis.S. Rajakarunakaran (6) considered a centrifugal pumping rotary system where the fault detection model was developed by using two different artificial neural network approaches which included feed forward network with back propagation algorithm and binary adaptive resonance network (ART1).Enrico Zio (7) in his paper developed a Neuro Fuzzy approach for pattern classification. J. Rafiee (8) presented a gear fault identification system using genetic algorithm (GA) and artificial neural networks (ANNs).Gang Niu (9) proposed a data-fusion strategy where vibration signals were collected, trend features were extracted, normalized and sent into neural network for feature-level fusion.Karim Salahshoor (10) used distributed pattern of three adaptive Neuro-fuzzy inference system (ANFIS) classifiers for an industrial $440 \mathrm{MW}$ power plant steam turbine with once-through Benson Type boiler.Ilyes Khelf (11) 
accurate identified the defects in rotating machines, using the combination of pattern recognition and nondestructive testing techniques such as vibration analysis and its indicators.Dimitrios Kateris (12) in his paper presented the architecture of a diagnostic system for extended faults in bearings based on neural networks which highlighted the combined use of kurtosis and the line integral of the acceleration signal. Unbalance faults were identified through a data driven approach applied to a rotor dynamic test rig fitted with multiple discs by R.B. Walker (13). The process of automating the localization was achieved by using an artificial neural network (ANN).

\section{Features Extraction Of Signal:}

The Root Mean Square (RMS) value and the phase value of the vibration for each of the vibration readings in $\mathrm{X}$ and $\mathrm{Y}$ direction for Left and Right bearings has been extracted from vibration signal using the vibration analyser. Denoising of the vibration signature has been done using low pass filter where maximum allowable frequency was $30 \mathrm{Hertz}$. Above $30 \mathrm{Hertz}$, no frequency values were considered for this research. Fig 1 represents the Time Domain vibration signal and Fig 2 shows the RMS value of vibration which is calculated directly using the following formula.

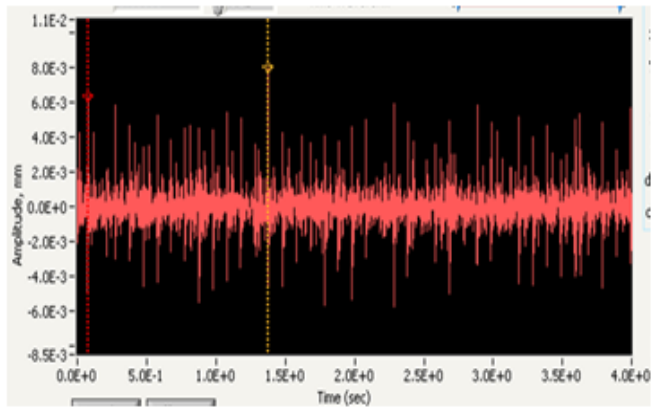

Fig 1: Time Domain Vibration Signal

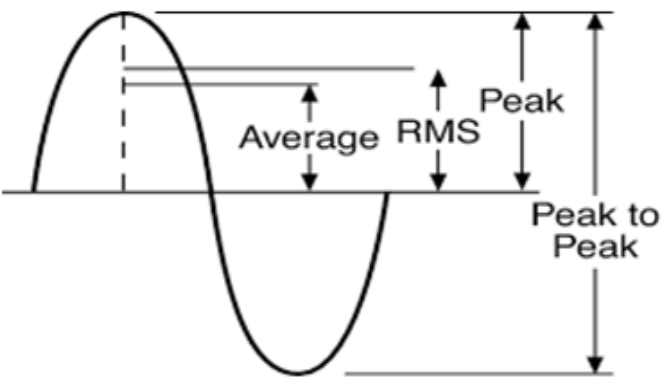

Fig 2: Features in Time Domain Signal

$\mathrm{RMS}=0.707 *$ peak value of the vibration signal

The peak value of the signal is obtained directly from the accelerometer attached in $\mathrm{X}$ and $\mathrm{Y}$ direction of left and right bearings.

\section{Basics of Artificial Neural Networking}

A neural network is a massively parallel distributed processor that has a natural tendency for storing knowledge and making it available for use. Artificial neural network (ANN) is a type of artificial intelligence, which has nonlinear information processing devices, built from interconnected elementary processing devices called neurons. Among all kinds of intelligent diagnosis methods, pattern recognition based on an Artificial Neural Network (ANN) has been widely used because of its power in self- organizing, unsupervised learning, and nonlinear pattern classification. The artificial neuron receives signals analogous to the natural electrochemical signals. The way information is processed and intelligence is stored depends on the architecture and algorithms of ANN. It is an online algorithm and receives data points one at a time. In this research work only one hidden layer has been used.

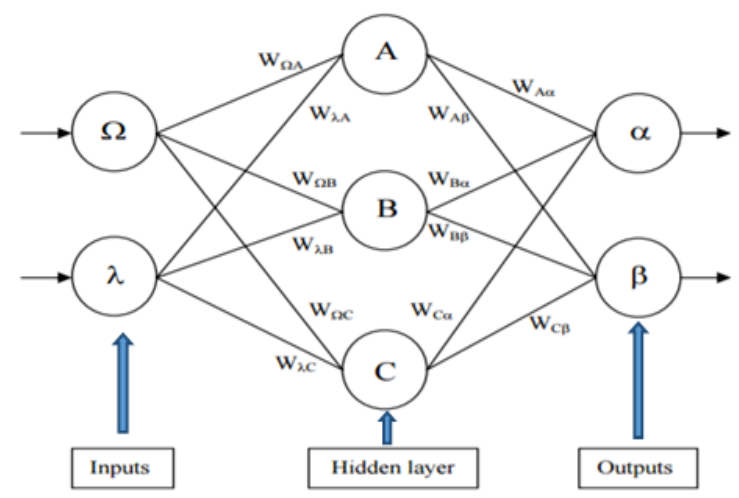

Fig 3: Typical multilayer feed forward neural network 
In the Fig $3 \Omega$ and $\lambda$ are the input neurons considered in the input layer. Neuron $A, B$, and $C$ are the hidden neurons in the hidden layer. $\alpha$ and $\beta$ are the output neurons. $\delta_{\alpha}$ and $\delta_{\beta}$ are the errors calculated. $\eta$ is the learning rate of the neurons.

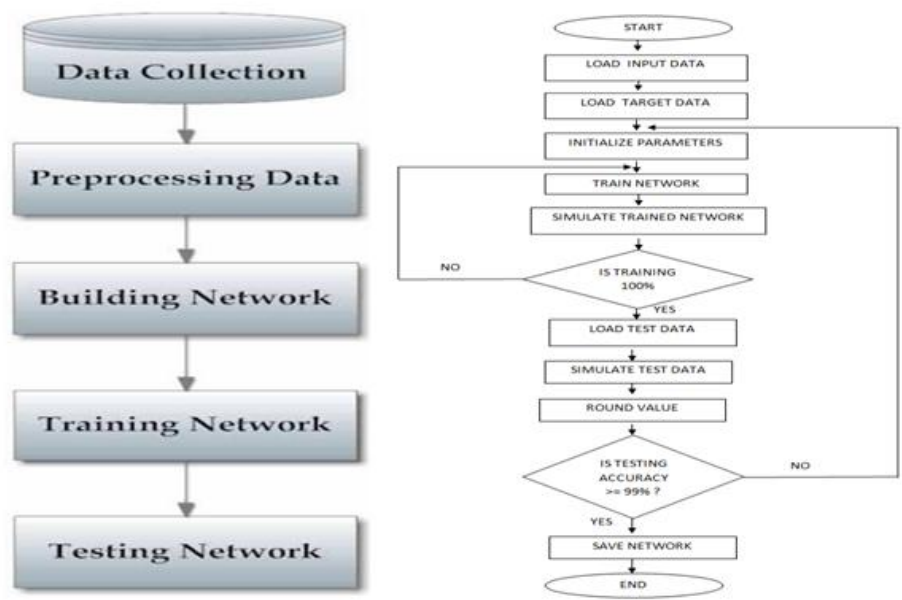

Fig 4: Flowchart of Artificial Neural Networking

Artificial Neural Networking is a very important tool used for prediction purposes. In this research, the ANN module has been developed and executed using JAVA programming code. Fig 4 clearly explains the step by step working of Artificial Neural Networking. The input layer consists of the values of unbalance mass (m), the eccentricity (e) and the rpm (r) at which the disc is rotating. Only one hidden layer has been considered with error as low as 0.0096.Value of $\eta$ has been kept as low 0.005 for the benefit of better convergence and accurate results. After the training of the neural network, the testing of the neural work is done for the determination of the unbalance plane.

4.1 Neural Network Calculations: Codes for calculation has been written in Java language and executed based on the network diagram as shown in Fig 3.

\section{Calculate errors of output neurons:}

$\delta_{\alpha}=$ out $_{\alpha}\left(1-\right.$ out $\left._{\alpha}\right)\left(\right.$ Target $_{\alpha}-$ out $\left._{\alpha}\right)$

$\delta_{\beta}=\operatorname{out}_{\beta}\left(1-\right.$ out $\left._{\beta}\right)\left(\right.$ Target $_{\beta}-$ out $\left._{\beta}\right)$

$\delta_{\alpha}$ and $\delta_{\beta}$ are theerrors of the output neurons which are calculated between the current output and the target output.

\section{Change output layer weights:}

$\mathrm{W}^{+}{ }_{\mathrm{A} \alpha}=\mathrm{W}_{\mathrm{A} \alpha}+\eta \delta_{\alpha}$ out $_{\mathrm{A}}$

$\mathrm{W}^{+}{ }_{\mathrm{A} \beta}=\mathrm{W}_{\mathrm{A} \beta}+\eta \delta_{\beta}$ out $_{\mathrm{A}}$

$\mathrm{W}_{\mathrm{B} \alpha}^{+}=\mathrm{W}_{\mathrm{B} \alpha}+\eta \delta_{\alpha}$ out $_{\mathrm{B}}$

$\mathrm{W}^{+}{ }_{\mathrm{B} \beta}=\mathrm{W}_{\mathrm{B} \beta}+\eta \delta_{\beta}$ out $_{\mathrm{B}}$

$\mathrm{W}^{+}{ }_{\mathrm{C} \alpha}=\mathrm{W}_{\mathrm{C} \alpha}+\eta \delta_{\alpha}$ out $_{\mathrm{C}}$

$\mathrm{W}^{+}{ }_{\mathrm{C} \beta}=\mathrm{W}_{\mathrm{C} \beta}+\eta \delta_{\beta}$ out $_{\mathrm{C}}$

The output layer weights are calculated as per the equation (iii) to equation (viii). $\mathrm{W}_{\mathrm{A} \alpha}, \mathrm{W}_{\mathrm{A} \beta}, \mathrm{W}_{\mathrm{B} \alpha}, \mathrm{W}_{\mathrm{B} \beta}, \mathrm{W}_{\mathrm{C} \alpha}$, $\mathrm{W}_{\mathrm{C} \beta}$ are the weights of the hidden layers. $\delta_{\alpha}$ and $\delta_{\beta}$ values are obtained from equation (i) and (ii). The learning rate $\eta$ is initially fixed at a very low value of 0.01 .

\section{Calculate (back-propagate) hidden layer errors}

$\delta_{\mathrm{A}}=\operatorname{out}_{\mathrm{A}}\left(1-\mathrm{out}_{\mathrm{A}}\right)\left(\delta_{\alpha} \mathrm{W}_{\mathrm{A} \alpha}+\delta_{\beta} \mathrm{W}_{\mathrm{A} \beta}\right)$

$\delta_{\mathrm{B}}=\operatorname{out}_{\mathrm{B}}\left(1-\right.$ out $\left._{\mathrm{B}}\right)\left(\delta_{\alpha} \mathrm{W}_{\mathrm{B} \alpha}+\delta_{\beta} \mathrm{W}_{\mathrm{B} \beta}\right)$

$\delta_{\mathrm{C}}=$ out $_{\mathrm{C}}\left(1-\right.$ out $\left._{\mathrm{C}}\right)\left(\delta_{\alpha} \mathrm{W}_{\mathrm{C} \alpha}+\delta_{\beta} \mathrm{W}_{\mathrm{C} \beta}\right)$

$\mathrm{Eq}-(\mathrm{xi})$

$\delta_{\mathrm{A}}, \delta_{\mathrm{B}}$ and $\delta_{\mathrm{C}}$ are the hidden layer errors which have been calculated using the formulas as mentioned in equation (ix), (x) and (xi). The values of $\mathrm{W}_{\mathrm{A} \alpha}, \mathrm{W}_{\mathrm{B} \alpha}, \mathrm{W}_{\mathrm{C} \alpha}, \mathrm{W}_{\mathrm{A} \beta}, \mathrm{W}_{\mathrm{B} \beta}$ and $\mathrm{W}_{\mathrm{C} \beta}$ have been obtained from equation (iii), (iv), (v), (vi), (vii) and (viii) respectively. $\delta_{\alpha}$ and $\delta_{\beta}$ values are obtained from equation (i) \& (ii).

\section{Change hidden layer weights}

$\mathrm{W}_{\lambda \mathrm{A}}^{+}=\mathrm{W}_{\lambda \mathrm{A}}+\eta \delta_{\mathrm{A}} \mathrm{in}_{\lambda}$

$\mathrm{W}_{\Omega \mathrm{A}}^{+}=\mathrm{W}_{\Omega \mathrm{A}}^{+}+\eta \delta_{\mathrm{A}} \mathrm{in}_{\Omega}$

$\mathrm{W}_{\lambda \mathrm{B}}^{+}=\mathrm{W}_{\lambda \mathrm{B}}+\eta \delta_{\mathrm{B}} \mathrm{in}_{\lambda}$

Eq-(xiv)

$\mathrm{W}_{\Omega \mathrm{B}}^{+}=\mathrm{W}_{\Omega \mathrm{B}}^{+}+\eta \delta_{\mathrm{B}} \mathrm{in}_{\Omega}$

$\mathrm{Eq}-(\mathrm{xv})$ 
$\mathrm{W}_{\lambda \mathrm{C}}^{+}=\mathrm{W}_{\lambda \mathrm{C}}+\eta \delta_{\mathrm{C}} \mathrm{in}_{\lambda}$

Eq-(xvi)

$\mathrm{W}^{+}{ }_{\Omega \mathrm{C}}=\mathrm{W}^{+}{ }_{\Omega \mathrm{C}}+\eta \delta_{\mathrm{C}}$ in $_{\Omega}$

Eq- (xvii)

The hidden layer weights are adjusted following the equation (xii), (xiii), (xiv), (xv), (xvi) and (xvii). If the calculated error of the output neurons is more than the set (desired) error, the process is repeated as shown in Figure 4.

\section{Experimental Setup And Procedure Of Data Collection}

Considering the main cause of rotating machine vibration as unbalance, a test rig for experimental validation of the model based identification technique was built. Schematic representation of the experimental set has been shown in the figures below.
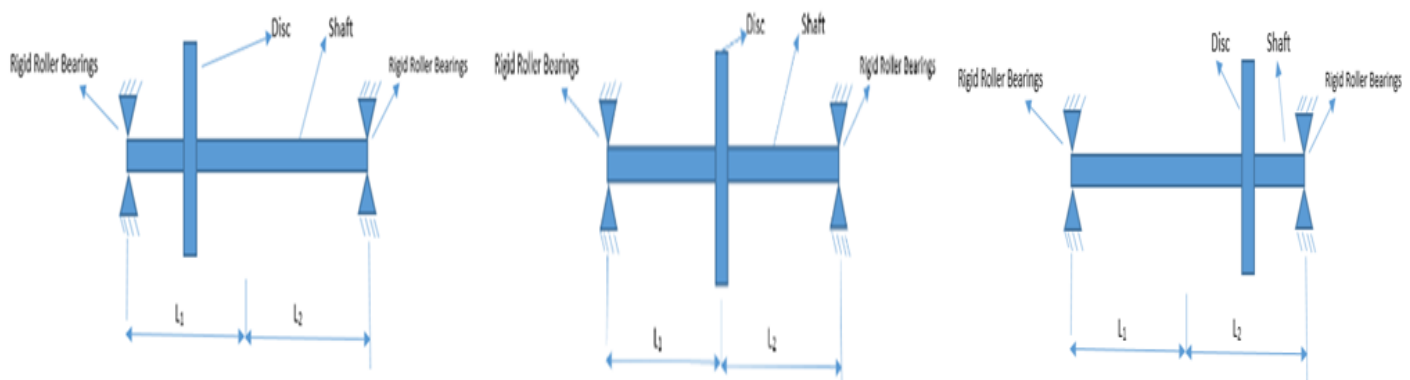

Figure 5:Schematic representation of the experimental set up (no fault case)
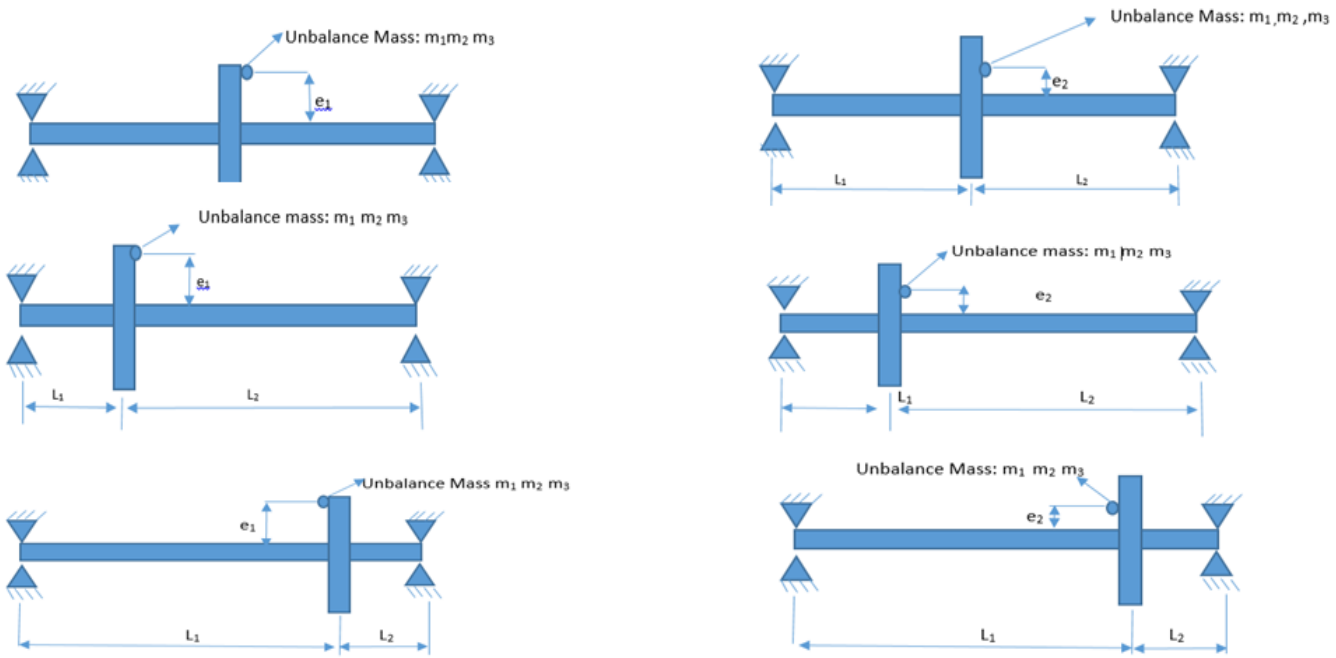

Figure6:Schematic representation of the experimental set up (with unbalance fault)

The experimental verification for the Unbalance Identification of mass on a shaft with single plane and two eccentricities has been performed on a Machine Fault Simulator provided at Central mechanical Engineering Research Institute (CSIR-CMERI) located at Durgapur. A rigid shaft considered to be massless is mounted between two roller bearings. The distance between the two bearings is $\mathrm{L}_{1}+\mathrm{L}_{2}$, which is $60 \mathrm{~cm}$. This shaft is connected to a Variable Frequency Drive (VFD) motor by a flexible coupling. To measure the vibration in X-Direction and Y-Direction at the two bearings, four accelerometers are connected; two in each bearing. The weight of the disc is 653 gram $\left(\mathrm{M}_{1}\right)$. The position of the disc is varied in three different locations: (i) $15 \mathrm{~cm}$ from left bearing (ii) $30 \mathrm{~cm}$ from left bearing which is the mid position on the shaft (iii) $45 \mathrm{~cm}$ from left bearing. Initially no unbalance mass was attached in order to get the no fault readings. Three unbalance masses of 8 gram $\left(\mathrm{m}_{1}\right), 12$ gram $\left(\mathrm{m}_{2}\right)$ and 16 gram $\left(\mathrm{m}_{3}\right)$ are attached subsequently to the disc at eccentricities $6.85 \mathrm{~cm}\left(\mathrm{e}_{1}\right)$ and $6.85 \mathrm{~cm}\left(\mathrm{e}_{2}\right)$ separately one by one. Then the shaft is rotated at rpm 300, 600, 900, 1200 and 1500; and simultaneously the vibration readings (RMS values) and their phase values in $\mathrm{x}$ and $\mathrm{y}$-direction at the two bearings were noted down. Artificial Neural Networking Techniques with varying training algorithms have been used to determine the unbalance plane of vibration.

Mass Unbalance: The unbalance masses used are 8 gram, 12 gram and 16 gram which are shown in the figures below. 


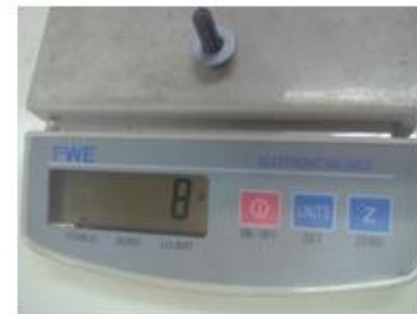

Fig 7

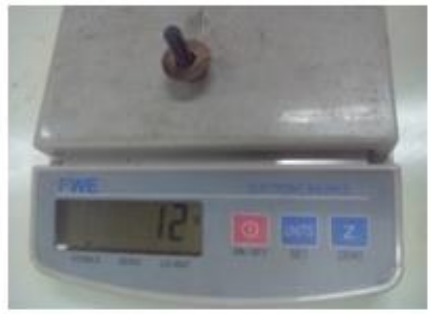

Fig 8

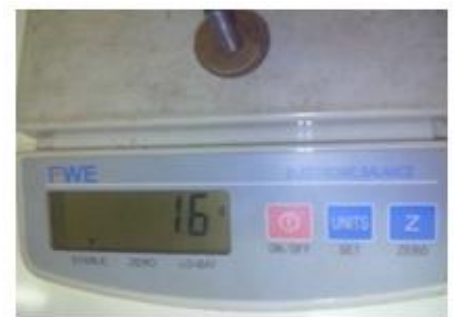

Fig 9

Fig 7: Unbalance mass of 8gram attached to the rotating system; Fig 8: Unbalance mass of 10gram attached to the rotating system; Fig 9: Unbalance mass of 8 gram attached to the rotating system

\section{Eccentricity:}

All rotors have some eccentricity. Eccentricity is present when geometrical center of the rotor and the mass center do not coincide along their length. In the present case, the disc is considered absolutely balanced where the Geometrical Centre of the disc coincides with the Centre of the Gravity of the disc. In the current research work eccentricity is the distance between the Geometrical Centre (Centre of rotation) and the points where the unbalance mass is to be attached. There are two locations at a distance of $6.85 \mathrm{~cm}$ from the centre of rotation $\left(\mathrm{e}_{1}\right)$ and at a distance of $4.85 \mathrm{~cm}$ from the centre of rotation of the disc $\left(\mathrm{e}_{2}\right)$.

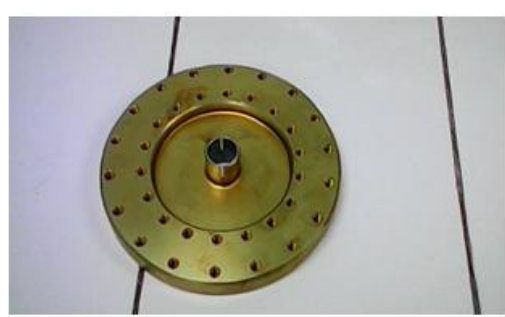

Fig 10: The rotating disc

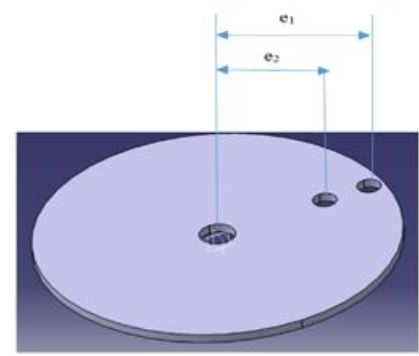

Fig 11: Schematicrepresentation of rotating disc

Plane of Unbalance:

The Figure 13, Figure 14 and Figure 15 shows the different position of the rotating disc placed on the shaft.

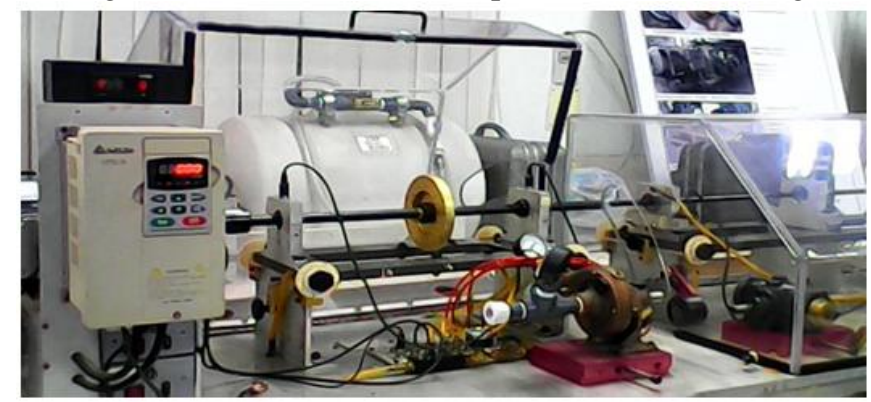

Fig 13: Machine Fault Simulator with rotating disc placed at centre position

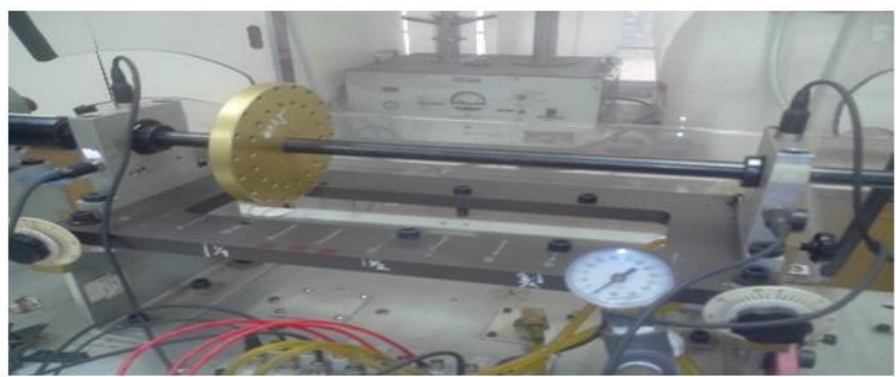

Fig 14: Machine Fault Simulator with rotating disc placed at left position 


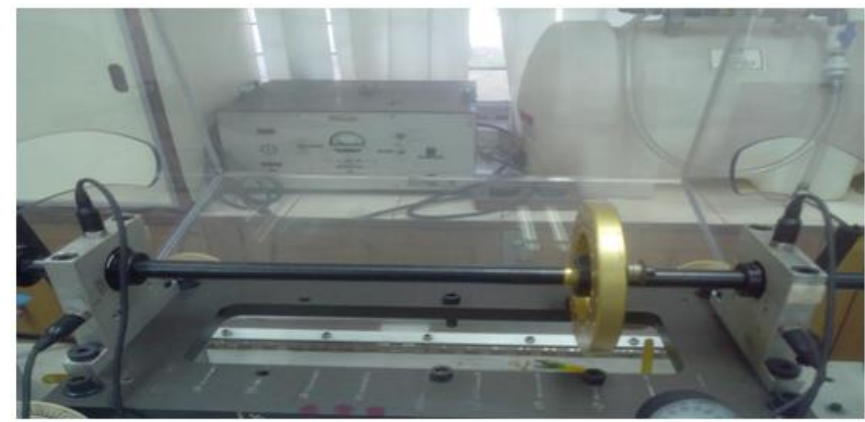

Fig 15: Machine Fault Simulator with rotating disc placed at right position

The design of experiment (Table 2) is based on the maximum possible combination of Unbalance mass (m), Eccentricity (e), Plane of unbalance (p) and the RPM(r) values. The table (Table 1) below provides the maximum and minimum values the independent parameters/input to the system.

Table 1: Independent/Input parameters and their levels

\begin{tabular}{|c|c|c|c|}
\hline Serial No & Independent /Input Parameters & Minimum Level & Maximum Level \\
\hline 1 & Unbalance Mass (m ) & $8 \mathrm{gram}$ & $16 \mathrm{gram}$ \\
\hline 2 & Eccentricity (e) & $4.85 \mathrm{~cm}$ & $6.85 \mathrm{~cm}$ \\
\hline 3 & Plane of unbalance (p) & $15 \mathrm{~cm}$ (measured from left bearing) & $45 \mathrm{~cm}$ (measured from left bearing) \\
\hline 4 & Revolutions per Minute (RPM) (r) & 300 & 1500 \\
\hline
\end{tabular}

Table 2: Design of Experiment

\begin{tabular}{|c|c|c|c|c|}
\hline Set & Experimental Codes & Unbalance Mass & Eccentricity & Location Of Unbalance Plane (In Cm) \\
\hline & & (IN GRAM) & $($ IN CM) & (FROM LEFT BEARING) \\
\hline 1 & NFL & 0 & 0 & 30 \\
\hline 2 & NFC & 0 & 0 & 45 \\
\hline 3 & NFR & 0 & 0 & 15 \\
\hline 4 & L8e2 & 8 & 4.85 & 30 \\
\hline 5 & C8e2 & 8 & 4.85 & 45 \\
\hline 6 & R8e2 & 8 & 6.85 & 15 \\
\hline 7 & L8e1 & 8 & 6.85 & 30 \\
\hline 8 & C8e1 & 8 & 6.85 & 45 \\
\hline 9 & R8e1 & 8 & 4.85 & 15 \\
\hline 10 & L12e2 & 12 & 4.85 & 30 \\
\hline 11 & C12e2 & 12 & 4.85 & 45 \\
\hline 12 & R12e2 & 12 & 6.85 & 15 \\
\hline 13 & L12e1 & 12 & 6.85 & 30 \\
\hline 14 & C12e1 & 12 & 6.85 & 45 \\
\hline 15 & R12e1 & 12 & 4.85 & 15 \\
\hline 16 & L16e2 & 16 & 4.85 & 30 \\
\hline 17 & C16e2 & 16 & 4.85 & 45 \\
\hline 18 & R16e2 & 16 & 6.85 & 15 \\
\hline 19 & L16e1 & 16 & 6.85 & 30 \\
\hline 20 & C16e1 & 16 & 6.85 & 45 \\
\hline 21 & R16e1 & 16 & & \\
\hline
\end{tabular}

The design of experiment as mentioned in the table 2 has been performed for 300, 600, 900, 1200 and 1500 RPM`s. In total there are 105 set of experiments.

\subsection{Indicators Used In Experiment :}

NFC: No fault disc at Center, NFL: No fault disc at Left position, NFR: No fault disc at Right position The number succeeding NFC, NFL, NFR represents the rpm at which the disc is rotating without any unbalance mass attached. With unbalance mass attached, the experimental code is written in the following format as mentioned below one after the other.

Position of Rotating Disc (p) Unbalance Mass (m)Eccentricity (e)Revolutions Per Minute (RPM) (r)

For example:C16e11500 represents the rotating disc placed at centre of the shaft with mass unbalance of 16 gram placed at an eccentricity $\mathrm{e}_{1}$ rotated at 1500 RPM. It is similar for rest of the experimental cases.

\section{Results And Discussion}

The input layer consists of the values of unbalance mass (m), Eccentricity (e), RPM ( r), RMS value of Left Bearing X-direction vibration LX (RMS), Phase value of Left Bearing X-direction vibration LX 
(Phase), RMS value of Left Bearing Y-direction vibration LY (RMS), Phase value of Left Bearing Y-direction vibration LY(Phase), RMS value of Right Bearing X-direction vibration RX (RMS), Phase value of Right Bearing X-direction vibration RX (Phase), RMS value of Right Bearing Y-direction vibration RY(RMS) and Phase value of Right Bearing Y-direction vibration RY(Phase)being fed to it .The output layer consists of the Plane of Unbalance (p) which has to be predicted through the online software. In between the input layer consisting of the input neurons and the output layer consisting of the output neurons lies the hidden layer where the calculation of weight adjustment and error minimization is done. Initially, the RMS and phase values in X and Y direction of left and right bearing has to be entered into the software. Weight of the neurons as well as the bias was fixed and entered thorough the input layer .Normalization was done for the input neurons and the output values are calculated using the formulas mentioned in the equations (i)-(xvii). Fig 16 and Fig 17 represents the initial GUI (Graphic User Interface)screens before the neural network is activated. The Fig 18. below represents the training of the neural networking which has been coded and developed using the equations from (i)-(xvii) in JAVA software. The training function used here is the Levenberg-Marquardt backpropagation with TANSIG as the transfer function. "Gradient Descent algorithm with momentum" has been used to minimize the error as an adaptive learning technique.

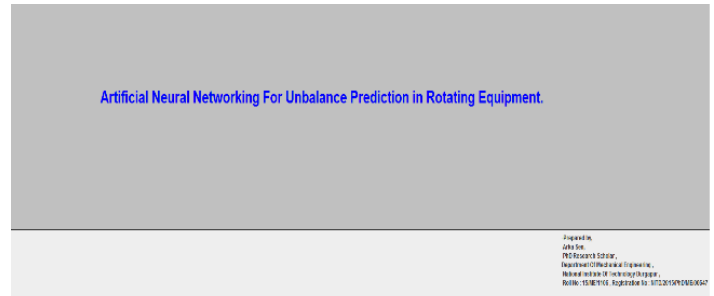

Fig 16: Initial Opening GUI Screen for the Online Software

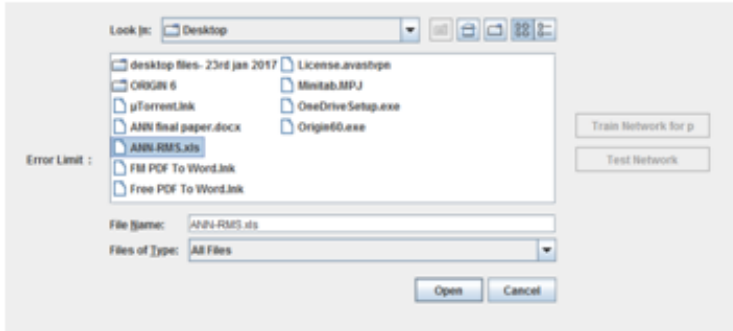

Fig 17: GUI Screen to read the values of RMS and Phase

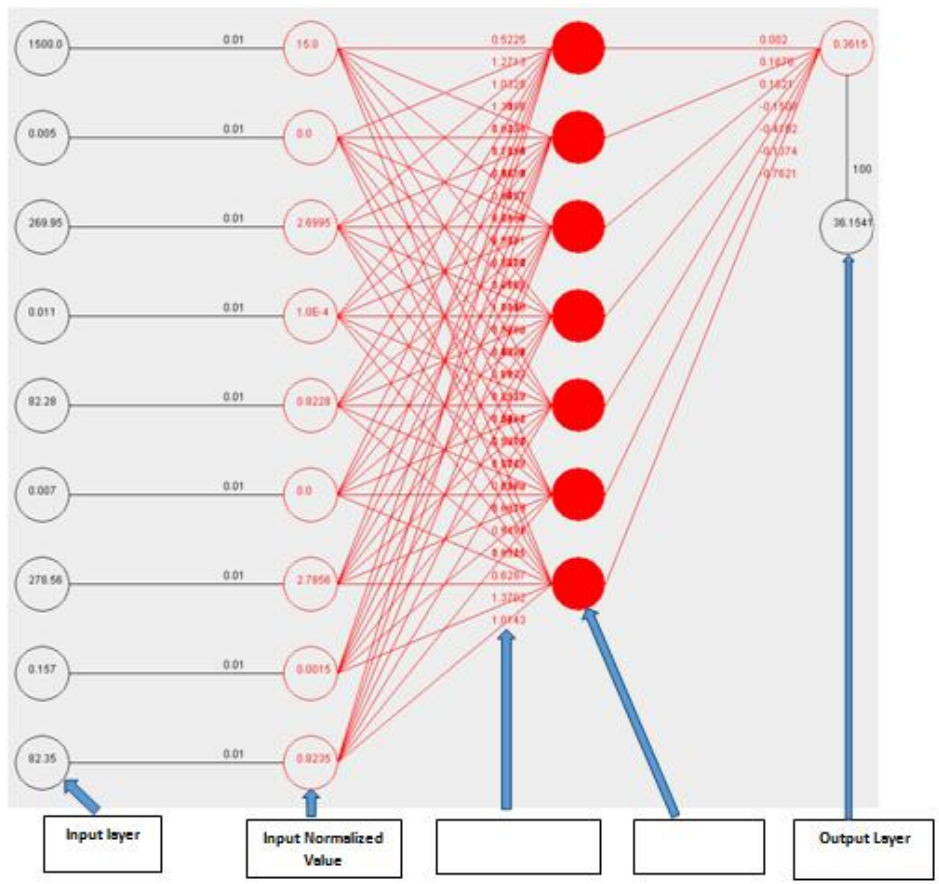

Fig18: Neural Networking Training user interface screen (for RMS and Phase) developed by JAVA software 


\subsection{Testing Of Artificial Neural Network for Unbalance Plane Detection:}

To test the artificial neural network for determination of the unbalance plane of vibration, the following data were given as input. For testing the neural network, the screen as shown in Fig 19 will appear on the screen.
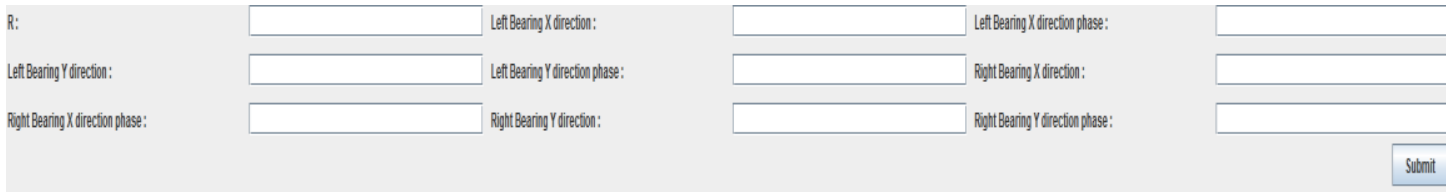

Fig19: User interface screen for online determining the unbalance plane of vibration

Input Parameters: The parameters provided for testing neural network are given below:

r (RPM): 1500

LX (RMS):0.0053LX (PHASE):269.95LY (RMS):0.010637LY (PHASE): 82.28

RX (RMS):0.00737RX (PHASE):278.56RY (RMS):0.015739RY (PHASE): 82.35

After entering these data as the input, the output is as follows, which is directly giving the unbalance plane of vibration as shown in Fig 20.

Predicted Output Using Online Software Prediction:

In the figure below, the unbalance plane of vibration is shown directly as the output of the process.

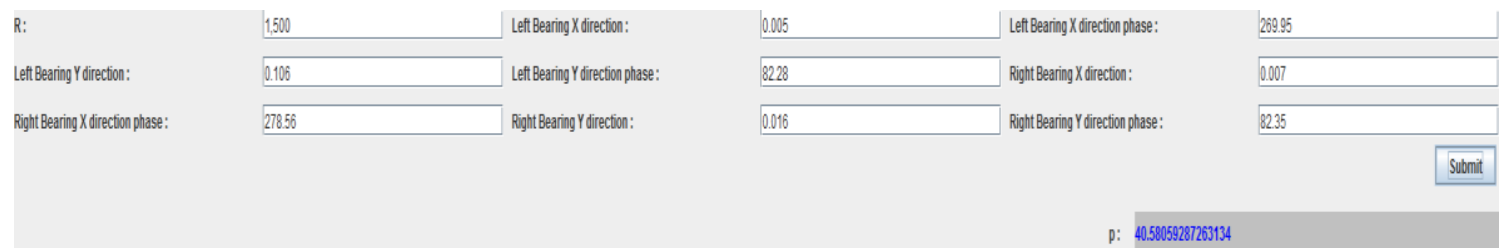

Fig 20:Unbalance Plane (p) Online Prediction User Interface Screen

Unbalance Plane Detection by the online software: $40.53 \mathrm{~cm}$ measured from left bearing

Experimental Unbalance: $45 \mathrm{~cm}$ measured from left bearing

Error: 4.470

Error \%: $9.93 \%$

\subsection{Prediction of Plane of Vibration using NNTOOL IN MATLAB:}

Inputs: The inputs to the MATLAB nntool bar are the Mass unbalance (m), Eccentricity (e), RPM (r), RMS value of Left Bearing X-direction vibration (LX), Phase value of Left Bearing X-direction vibration LX (Phase), RMS value of Left Bearing Y-direction vibration (LY), Phase value of Left Bearing Y-direction vibration LY(Phase), RMS value of Right Bearing X-direction vibration(RX), Phase value of Right Bearing Xdirection vibration RX (Phase), RMS value of Right Bearing Y-direction vibration (RY) and Phase value of Right Bearing Y-direction vibration RY(Phase).The two Adaptive Learning Functions available in Matlab are the LEARNGDM - Gradient Descent with momentum and LEARNGD - Gradient Descent. The transfer functions available are: LOGSIG - Log Sigma, PURELIN - Pure Linear and TANSIG - Tan Sigma. There are 12 (twelve) different types of training algorithms available in Matlab for performing the training of the neural network which have been analysed in details below.

\subsubsection{Comparison of outputs using different Training Function in Neural Networking:}

LEARNGDM - Gradient Descent with Momentum has been used as an "Adaptive Learning Function" for prediction of the unbalance plane. The transfer function used in MATLAB is TANSIG. Comparison of results for various Training algorithms used in Neural Network has been shown from Fig 21 to Fig 32. These figures explains the performance of the neural network using MATLAB. It has been observed that in most of the cases the training validation and the test data coincide at a point where the Mean Square error value is very low. Also the performance graph in most cases indicate steady slope between the training and the testing data which validates the point of convergence.

Inputs to the neural network are:

r (RPM): 1500

LX (RMS): 0.0053LX (PHASE):269.95LY (RMS):0.010637LY (PHASE):82.28

RX (RMS): 0.00737 RX (PHASE):278.56RY (RMS):0.015739RY (PHASE):82.35 
Output: Plane of Unbalance (p). Comparison table for the different the unbalance plane as calculated by different training methods is as follows:
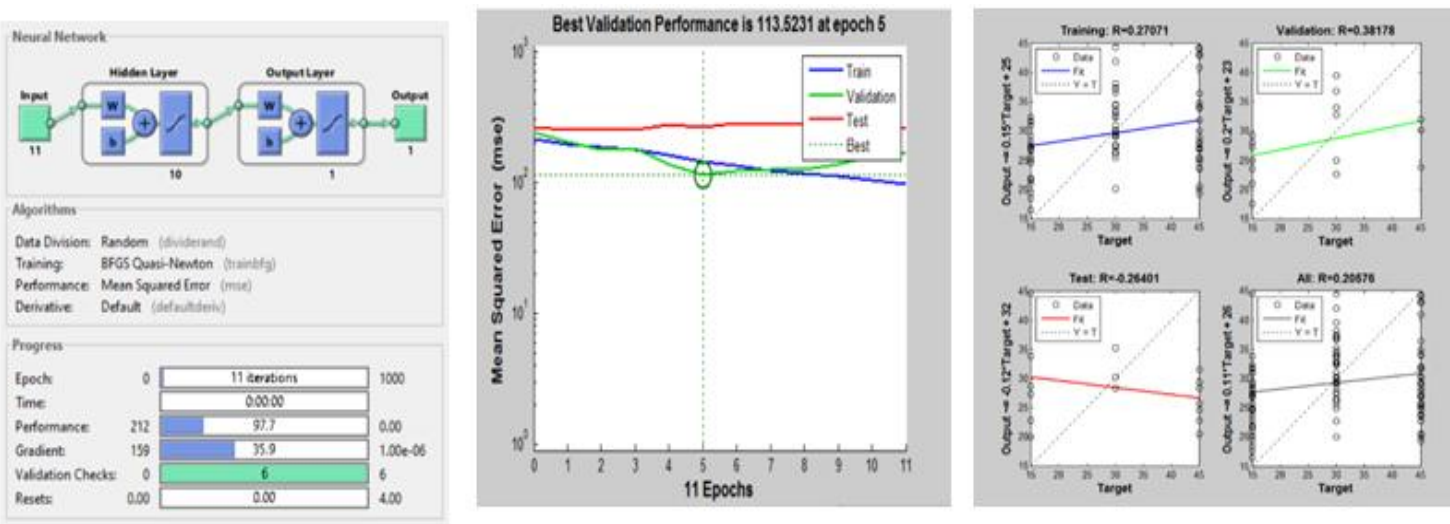

Fig 21: Neural network by TRAINBFG (BFGS quasi-Newton backpropagation)
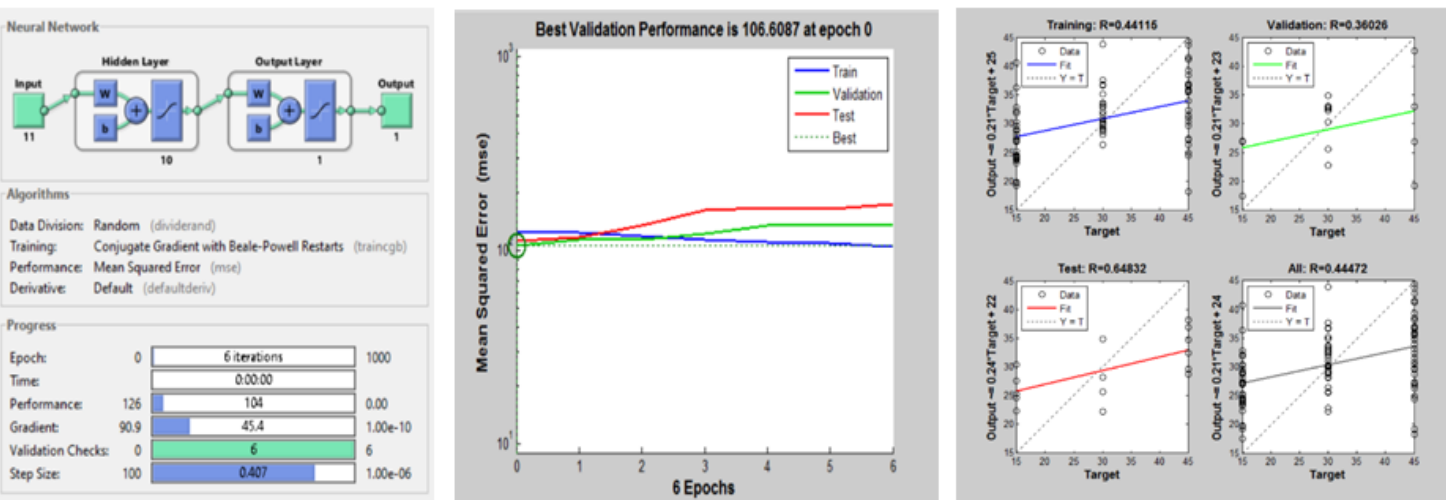

Fig 22: Neural network by TRAINCGB (Powell -Beale conjugate gradient backpropagation)
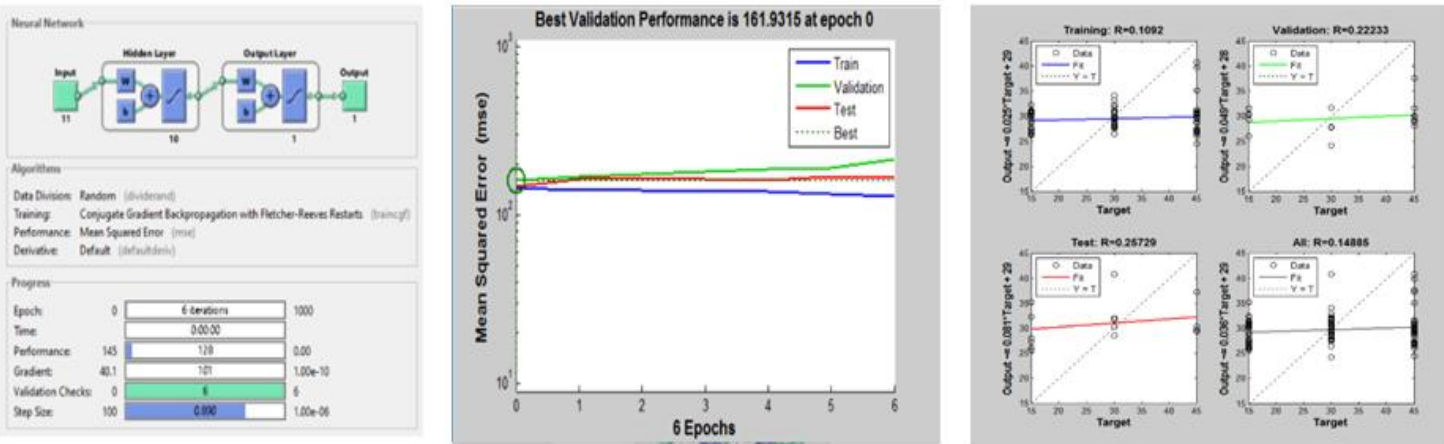

Fig 23: Neural network by TRAINCGF (Fletcher-Powell conjugate gradient backpropagation)
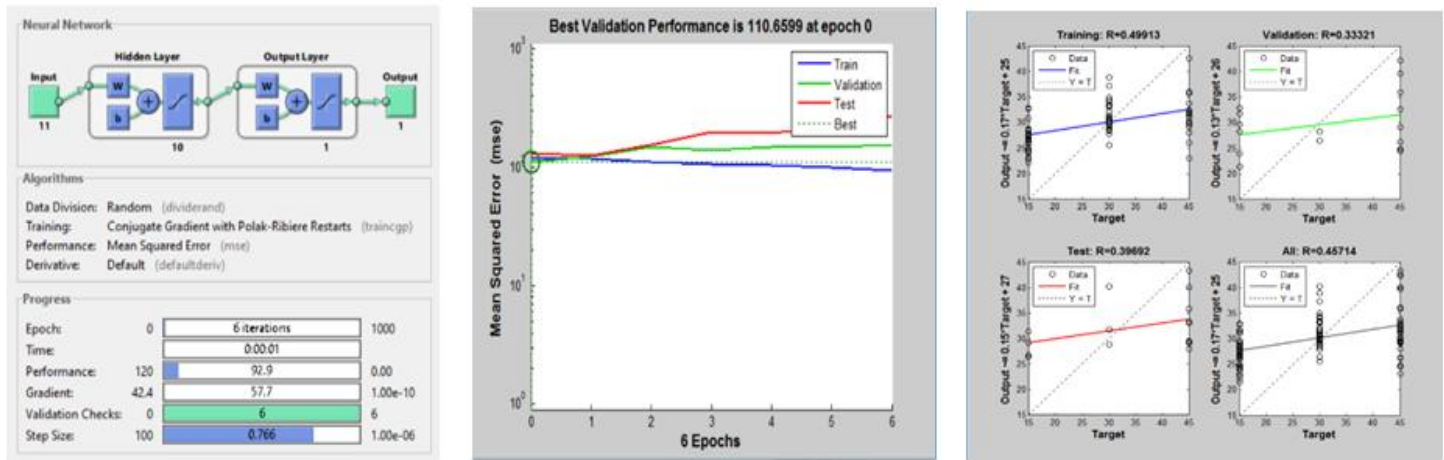

Fig 24: Neural network by TRAINCGP (Polak-Ribiere conjugate gradient backpropagation) 

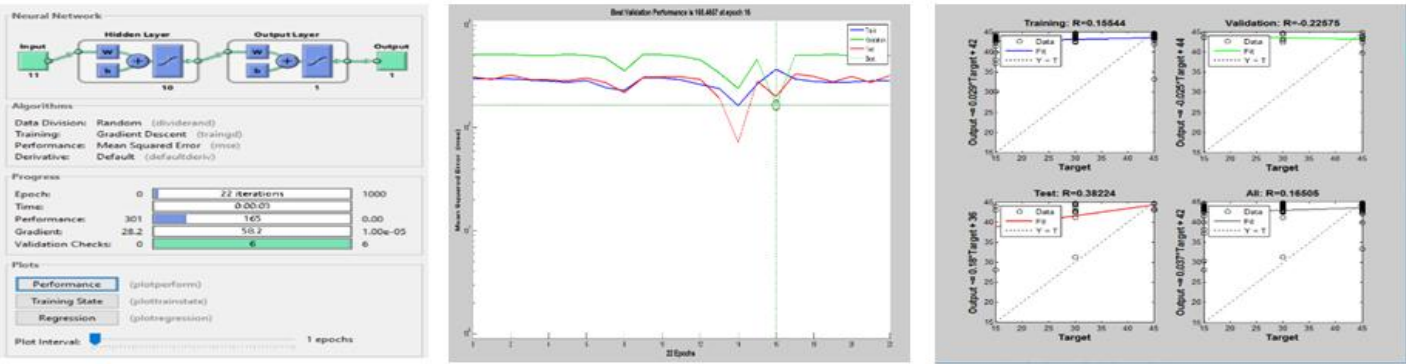

Fig 25: Neural network by TRAINGD (Gradient descent backpropagation)
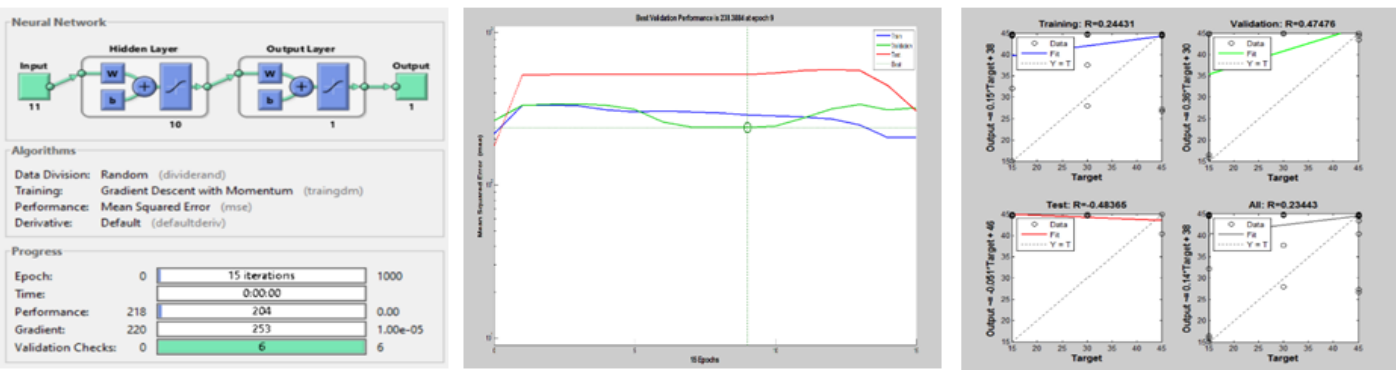

Fig 26: Neural network by TRAINGDM (Gradient descent with momentum backpropagation)
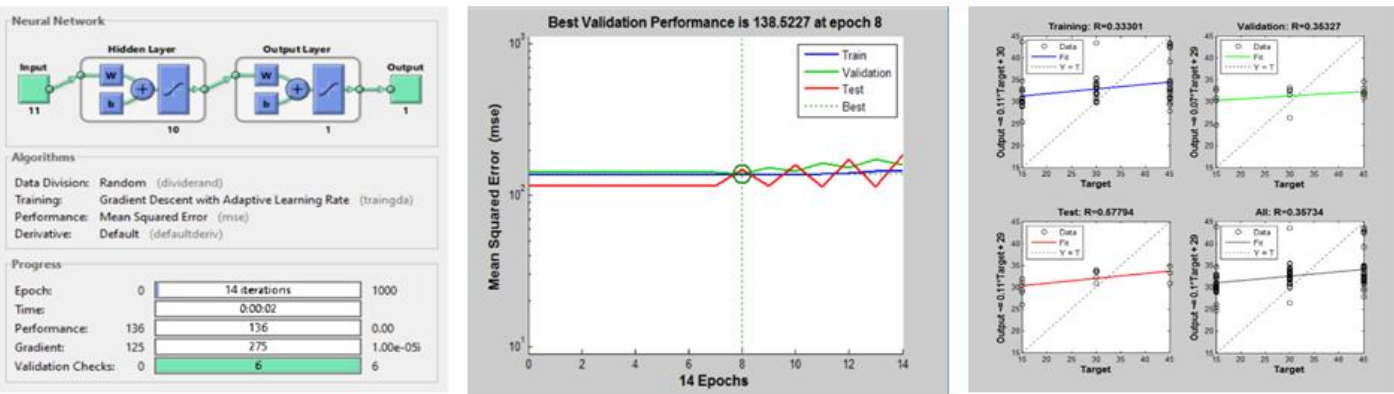

Fig 27: Neural network by TRAINGDA (Gradient descent with adaptive lr backpropagation)
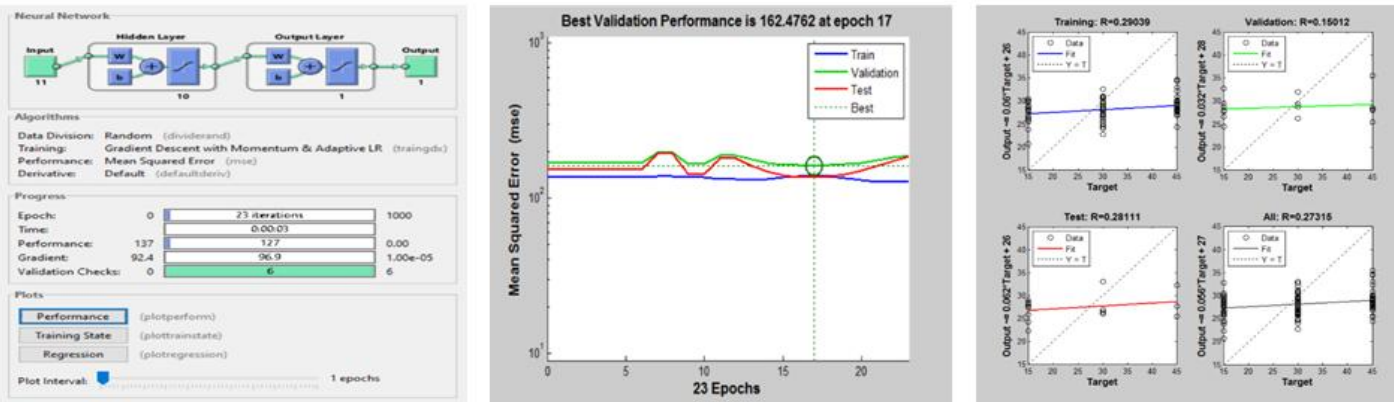

Fig 28: Neural network by TRAINGDX (Gradient descent w/momentum \& adaptive lr backpropagation)
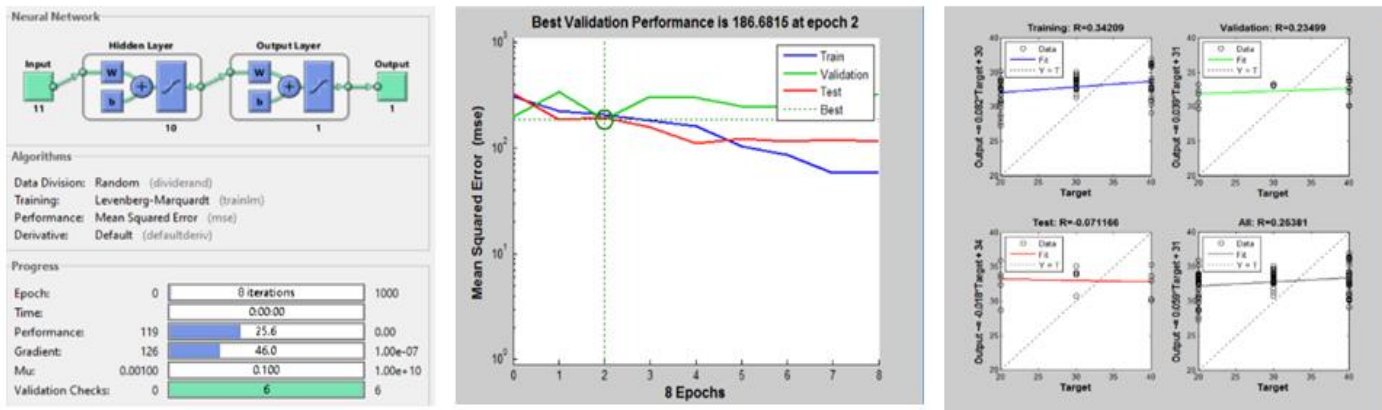

Fig29: Neural network by TRAINLM (Levenberg-Marquardt backpropagation) 

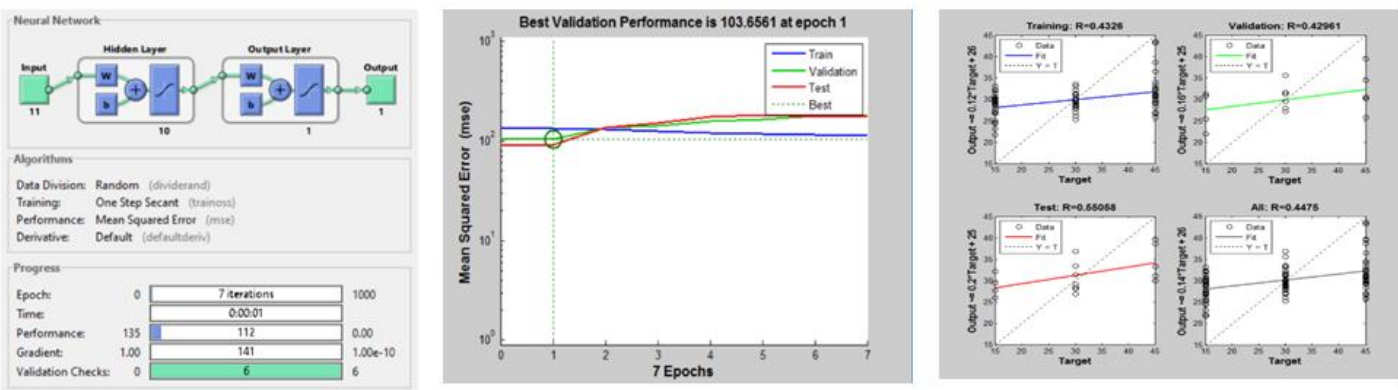

Fig30: Neural network by TRAINOSS (One step secant backpropagation)
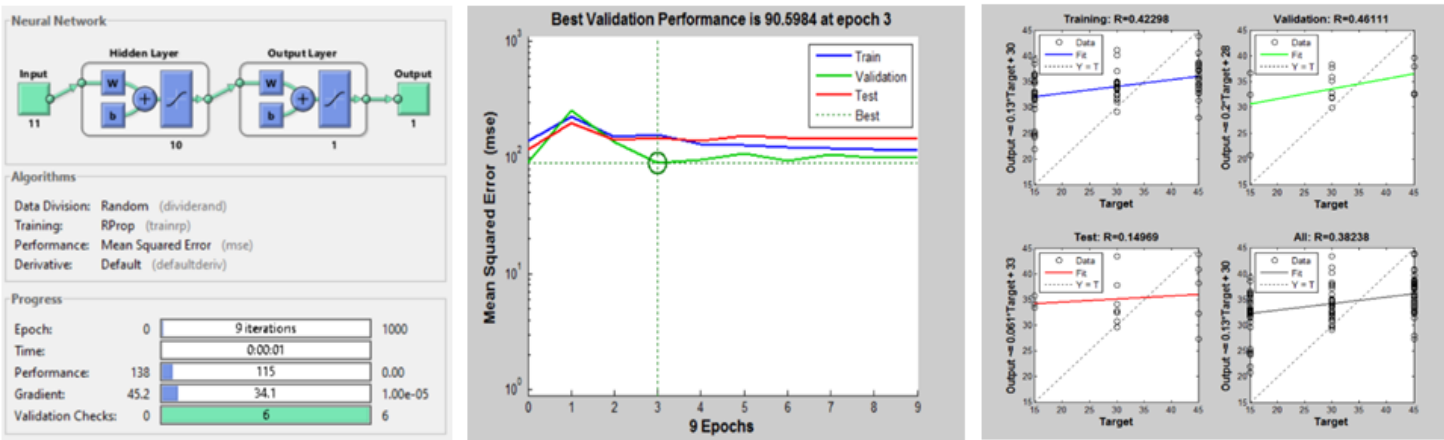

Fig31: Neural network by TRAINNRP (Resilient backpropagation (Rprop))
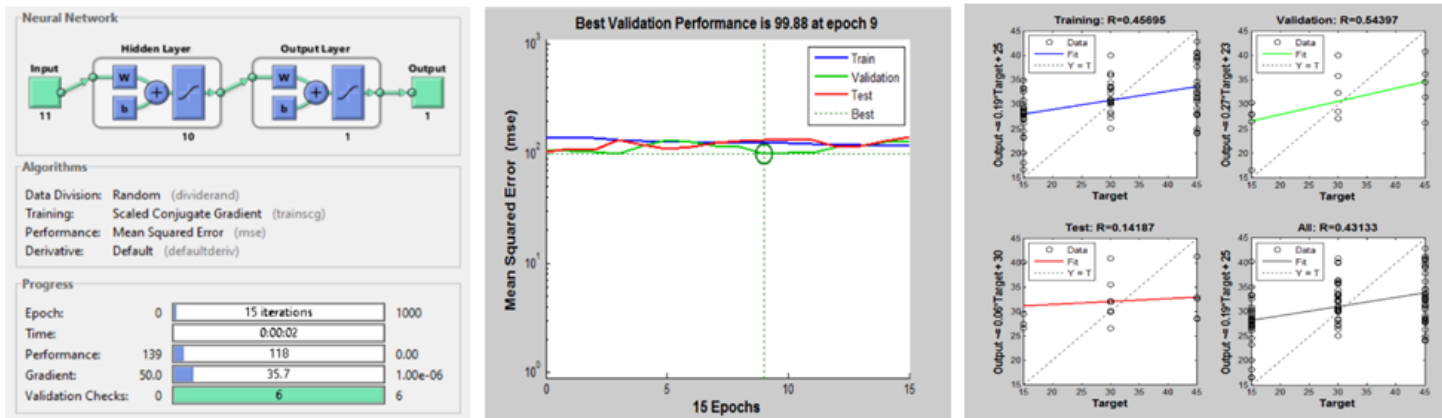

Fig32: Neural network by TRAINSCG (Scaled conjugate gradient backpropagation)

A comparative analysis of results as obtained from neural networks with different training algorithms and their errors have been summarized in the Table 4 as shown below.

Table 4: Comparative Analysis of Result (Training Function GRADIENT DESCENT WITH MOMENTUM)

\begin{tabular}{|c|c|c|c|c|}
\hline Methodology & $\begin{array}{c}\text { Actual Plane Of } \\
\text { Unbalance }\end{array}$ & $\begin{array}{c}\text { Predicted Plane Of } \\
\text { Unbalance }\end{array}$ & Error & Error (In \%) \\
\hline $\begin{array}{c}\text { ONLINE SOFTWARE } \\
\text { (using java code) }\end{array}$ & 45 & 40.53 & 4.470 & 9.93 \\
\hline $\begin{array}{c}\text { BFGS quasi-Newton } \\
\text { backpropagation }\end{array}$ & 45 & 44.1519 & 0.8481 & 1.88 \\
\hline $\begin{array}{c}\text { Powell -Beale conjugate } \\
\text { gradient backpropagation }\end{array}$ & 45 & 43.8034 & 1.1966 & 2.65 \\
\hline $\begin{array}{c}\text { Fletcher-Powell conjugate } \\
\text { gradient backpropagation }\end{array}$ & 45 & 39.7941 & 5.2059 & 11.56 \\
\hline $\begin{array}{c}\text { Polak-Ribiere conjugate gradient } \\
\text { backpropagation }\end{array}$ & 45 & 43.334 & 1.666 & 3.70 \\
\hline $\begin{array}{c}\text { Gradient descent } \\
\text { backpropagation }\end{array}$ & 45 & 44.8371 & 0.1629 & 0.362 \\
\hline $\begin{array}{c}\text { Gradient descent with } \\
\text { momentum backpropagation }\end{array}$ & 45 & 44.9959 & .0041 & .0091 \\
\hline $\begin{array}{c}\text { Gradient descent with adaptive } \\
\text { lr backpropagation }\end{array}$ & 45 & 42.9352 & 2.0648 & 4.588 \\
\hline $\begin{array}{c}\text { Gradient descent w/momentum } \\
\& \text { adaptive lr backpropagation }\end{array}$ & 45 & 35.5315 & 9.4685 & 21.0411 \\
\hline $\begin{array}{c}\text { Levenberg-Marquardt } \\
\text { backpropagation }\end{array}$ & 45 & 39.15 & 5.85 & 13 \\
\hline
\end{tabular}




\begin{tabular}{|c|c|c|c|c|}
\hline One step secant backpropagation & 45 & 39.5148 & 5.4852 & 12.18 \\
\hline Resilient backpropagation & 45 & 43.8263 & 1.1764 & 2.614 \\
\hline $\begin{array}{c}\text { Scaled conjugate gradient } \\
\text { backpropagation }\end{array}$ & 45 & 41.287 & 3.713 & 8.25 \\
\hline
\end{tabular}

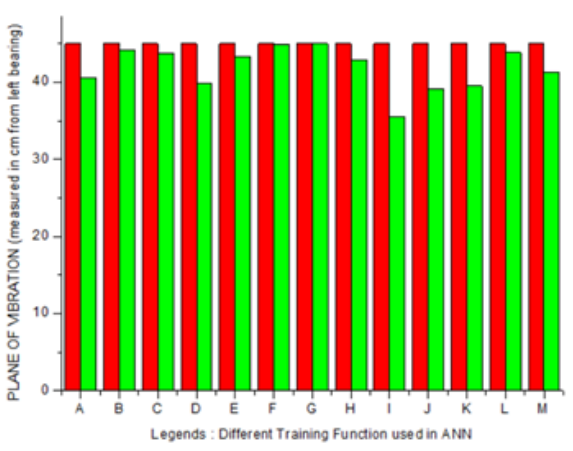

Fig 33:Actual vs Prediction Unbalance Plane Histogram

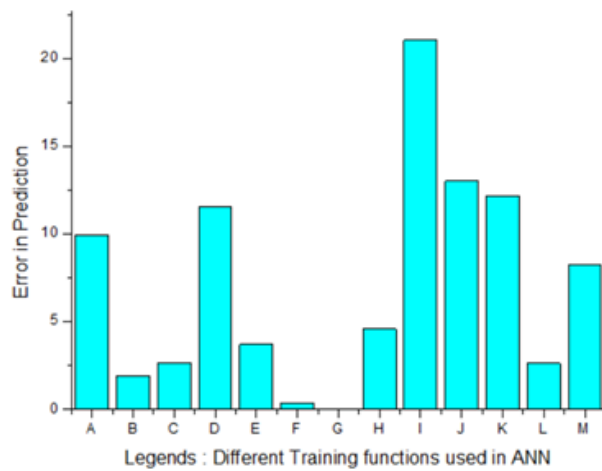

Fig 34: Error Histogram

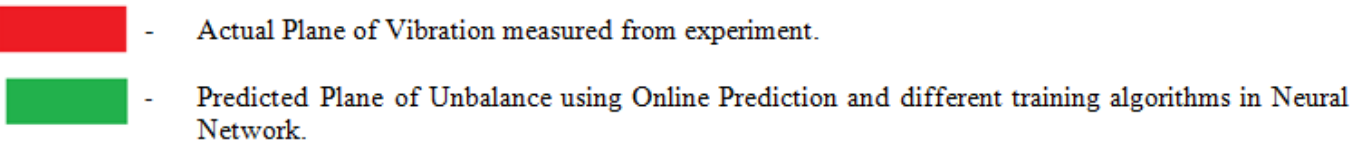

Legends used in Fig 33 and Fig 34:

ONLINE PREDICTION - Represented by " $A$ " in Histogram,

TRAINBHG - Represented by "B" in Histogram,

TRAINCGB - Represented by "C" in Histogram,

TRAINCGF - Represented by "D" in Histogram,

TRAINCGP - Represented by "E" in Histogram,

TRAINGD - Represented by " $F$ " in Histogram,

TRAINGDM- Represented by "G" in Histogram,

TRAINGDA - Represented by " $\mathrm{H}$ " in Histogram,

TRAINGDX- Represented by "I" in Histogram,

TRAINLM - Represented by "J" in Histogram,

TRAINOSS - Represented by " $K$ " in Histogram,

TRAINNRP -Represented by "L" in Histogram,

TRAINSCG - Represented by " $M$ " in Histogram.

Fig 33 and Fig 34 represents the histogram for the unbalance plane of vibration and the error histogram respectively. From these two figures it was evident that the "Gradient descent with momentum training algorithm" has the least error for prediction.

\section{Conclusion And Future Scope Of Work:}

In this research work the unbalance plane of vibration has been detected using the online software coded in Java and by the Matlab nntool. It has been noticed that the software did provide better accuracy of result than the MATLAB simulation with the application of nntool using Levenberg-Marquardt backpropagation training algorithm. Optimization of the weights and the learning rate (which has been coded within the software using JAVA application) was done and the error was below the MATLAB predicted error. Unlike in MATLAB where the Input, Target and Sample file has to be created separately for calculation, the values of RMS and phase can be directly entered into the Online Software and on clicking the submit button the software returns the predicted plane of unbalance. The only constrain in this online software is that it has to be fed with numerous number of experimental data for various cases and estimations before running the testing phase for the unbalance plane prediction and getting the accurate result.

It has also been concluded that, although the neural network can be trained with different types of training algorithms (in this research paper 12 different types of training algorithms have been used ), the best result comes with the Gradient Descent with Momentum where the prediction is almost near to the actual value. The next Training algorithm with minimum error is the Gradient Descent algorithm. It is clearly seen that Levenberg-Marquardt backpropagation used in MATLAB nntool gives 13\% error where the same training function when used in ONLINE Software yielded error of $9.93 \%$. Thus it can be concluded that, using 
Levenberg-Marquardt backpropagation training function, the ONLINE software is a better choice as compared to MATLAB nntool. In the future scope of the work, the ONLINE software shall be updated with training algorithms like "Gradient descent with Momentum" and "Gradient Descent" to provide better accuracy in prediction.

\section{Acknowledgement}

The author is thankful to the Department of Mechanical Engineering, National Institute of Technology Durgapur and Condition Monitoring Department of Central Mechanical Engineering Research Institute located at Durgapur to help me to carry out this research work on the "Application of Artificial Neural Networking for Determining the Plane of Vibration in Rotating System".

\section{References}

[1]. Yazhao Qiu, Singiresu S. Rao ,A fuzzy approach for the analysis of unbalanced nonlinear rotor systems , Journal of Sound and Vibration 284 (2005) 299-323.

[2]. Jiangping Wang, Hongtao Hu, Vibration-based fault diagnosis of pump using fuzzy technique, Measurement 39 (2006) 176-185

[3]. Javier Sanz, Ricardo Perer, Consuelo Huert,Fault diagnosis of rotating machinery based on auto-associative neural networks and wavelet transforms,Journal of Sound and Vibration 302, (2007) 981-999.

[4]. Yaguo Lei, Zhengji He, YanyangZi, Qiao Hu, Fault diagnosis of rotating machinery based on multiple ANFIS combination with GAs, Mechanical Systems and Signal Processing 21 (2007) 2280-2294

[5]. Yaguo Lei, Zhengjia He, Yanyang Z,A new approach to intelligent fault diagnosis of rotating machinery,Expert Systems with Applications 35 (2008) 1593-1600

[6]. S. Rajakarunakaran, P. Venkumar, D. Devaraj, K. Surya Prakas Rao, Artificial neural network approach for fault detection in rotary system, Applied Soft Computing 8 (2008) 740-748.

[7]. Enrico Zio, Giulio Gola, A neuro-fuzzy technique for fault diagnosis and its application to rotating machinery, Reliability Engineering and System Safety 94 (2009) 78-88.

[8]. J. Rafiee, P.W. Tse , A. Harifi , M.H. Sadeghi, A novel technique for selecting mother wavelet function using an intelligent fault diagnosis system,Expert Systems with Applications 36 (2009) 4862-4875

[9]. [9]Gang Niu, Bo-Suk Yang, Intelligent condition monitoring and prognostics system based on data-fusion strategy,Expert Systems with Applications 37 (2010) 8831-8840.

[10]. Karim Salahshoor, Majid SoleimaniKhoshro, MojtabaKordestani,Fault detection and diagnosis of an industrial steam turbine using a distributed configuration of adaptive neuro-fuzzy inference systems,Simulation Modelling Practice and Theory 19 (2011) 12801293.

[11]. Ilyes Khelf, Lakhdar Laouar, Abdelaziz M.Bouchelaghem, Didier Rémond, Salah Saad, Adaptive fault diagnosis in rotating machines using indicators selection, Mechanical SystemsandSignalProcessing40(2013)452-468.

[12]. Dimitrios Kateris, Dimitrios Moshou, Xanthoula-EiriniPantazi, Ioannis Gravalos, Nader Sawalhi and Spiros Loutridis, A machine learning approach for the condition monitoring of rotating machinery, Journal of Mechanical Science and Technology 28 (1) (2014) $61 \sim 71$

[13]. R.B. Walker, R. Vayanat, S. Perinpanayagam, I.K. Jennions, Unbalance localization through machine nonlinearities using an artificial neural network approach Mechanism and Machine Theory 75 (2014) 54-66 